\title{
The Role of Black Soybean and Purple Sweet Potato Active Compound on Advanced Glycation End-Product in Streptozotocin-Induced Type 2 Diabetes Mellitus Rat
}

\author{
Abdul Gofur ${ }^{1 *}$, Siti Nur Arifah ${ }^{1}$, Yuslinda Annisa ${ }^{1}$, Agung Witjoro ${ }^{1}$, Mochammad Fitri Atho'illah ${ }^{2}$, \\ Sri Rahayu Lestari ${ }^{1}$
}

${ }^{1}$ Department of Biology, Universitas Negeri Malang, Malang 65145, Indonesia

2 Department of Biology, Brawijaya University, Malang 65145, Indonesia

Article history:

Submission January 2019

Revised February 2019

Accepted March 2019

*Corresponding author:

E-mail: abdul.gofur.fmipa@um.ac.id

\begin{abstract}
Diabetic nephropathy is one of the diabetes complications attacking kidney leading to kidney damage. Hyperglycemia accompanying DM causes the increase of Advanced Glycation End-Product (AGE) and Receptor Advanced Glycation EndProduct (RAGE) activity, then develop kidney damage and other diabetes complications. The study aimed to investigate the effect of black soybean, purple sweet potato, or their combination on the expression of AGE, RAGE, and kidney necrosis T2DM model rats. The rats were given with high-calorie diet for five weeks and then injected with a low dose of streptozotocin (30 m/kg Body Weight) in intraperitoneal. DM rats were divided into: normal, K- (T2DM), K+ (T2DM + glibenclamide $0.6 \mathrm{mg} / \mathrm{kg}$ body weight), P1 (T2DM + black soybean), P2 (T2DM + purple sweet potato), and P3-5 Combination 1-3 (T2DM + combination of black soybean and purple sweet potato in ratio of $1: 3,2: 2$, and $3: 1)$. DM rats were then given the treatments for thirty days. The effect of black soybean, purple sweet potato, or the combination of both was evaluated through the expression of AGE, RAGE, and necrosis of renal tubules. The changes in renal tubules histological characteristics were evaluated using hematoxylin-eosin (HE) staining. Immunohistochemistry analysis of renal tubules was to evaluate AGE-RAGE expression after the treatments. The research results indicated that there was a significant difference from the combination of black soybean and purple sweet potato in reducing AGE, RAGE, and renal tubules necrosis. The BSB and PSP combination ratio of 1:1 was able to improve renal tubules, decrease the expression of AGE and RAGE towards near normal. The combination of black soybean and purple sweet potato could be used as one of the alternatives to improve kidney damage in diabetic nephropathy.
\end{abstract}

Keywords: Black soybean, purple sweet potato, RAGE, AGE, renal tubules, T2DM

\section{Introduction}

Diabetes mellitus (DM) is a metabolic disorder which characterized by impaired of regulation in glucose, lipid, and protein metabolism [1]. DM is marker by high glucose level in blood stream [2]. Diabetic nephropathy (DN) is one of the most critical complications caused by DM with a consequence of kidney damage [3, 4]. DN is chronic kidney disease and has a high value of mortality among people with diabetes. DN has the most pa- tient, which is $30-40 \%$ of all T1DM and T2DM patients $[5,6]$. The increase in AGE-RAGE levels causes a histological change in the kidney [7]. DN marks with the occurrence of glomerular basement membrane thickening, the enlargement of extracellular matrix of mesangial cells, atrophy in tubules, the damage in microvascular vessels, and the presence of necrosis to fibrosis in the interstitial tubules $[3,6,8,9]$. The hyperglycemic condition that accompanies DM could cause an increase

\section{How to cite:}

Gofur A, Arifah SN, Annisa Y et al. (2019) The Role of Black Soybean and Purple Sweet Potato Active Compound on Advanced Glycation End-Product in Streptozotocin-Induced Type 2 Diabetes Mellitus Rat. Journal of Tropical Life Science 9 (2): 139 - 145. doi: 10.11594/jtls.09.02.02 
in the number of Advanced Glycation End-Product (AGE) $[5,8,9]$. AGE is formed due to the interaction between glucose and amino acid group (especially lysine and arginine residue) and fat in non-enzymatic [5, 9, 10]. AGE will be recognized by a specific receptor called receptor of AGE (RAGE). When the AGE level increases, RAGE will increase. During the hyperglycemic condition, AGE accumulation occurs causing damage in kidney and other diabetic complications [10].

The current diabetes mellitus treatment is less optimal, especially to cure kidney damage due to DM complications. Herbal medicine is an alternative supported by the WHO, especially to cure degenerative diseases, such as diabetes [11]. Soybean (Glycine max) is a plant from Fabaceae family (legume) and epidemiologically, it has various benefits for health. Black soybean is one of soybean (G. max) varieties. The plant contains isoflavones and anthocyanin that has anti-oxidant, anticancer, anti-diabetes, and anti-inflammation activities [12, 13]. Purple sweet potato (Ipomoea batatas) is generally used by the traditional community to consume its roots and leaves. I. batatas also contain anthocyanin that functions as alternative treatment for various diseases, especially degenerative diseases [14, 15]. The combination of black soybean and purple sweet potato reported have ameliorate effect to suppressing reactive oxygen species (ROS) to improve sperm quality [16]. ROS is free radicals that can cause various disease by increasing oxidative stress in the body [17].

However, the combination of black soybean and purple sweet potato as T2DM alternative treatment, primarily to treat kidney damage (diabetic nephropathy) is never done therefore. This research aimed to evaluate the combination of black soybean and purple sweet potato as an anti-diabetic nephropathy agent.

\section{Material and Methods \\ Black soybean and purple sweet potato prepara- tion}

Purple sweet potato (were harvested at \pm 4 months) obtained from purple sweet potato plantation in Kawi Mountain, Malang Regency. Black soybean (var. Detam-1, were harvested at \pm 3 months) purchased Indonesian Legumes and Tuber Crop Research Institute (ILETRI), Malang Regency. Purple sweet potato was washed, cut into small pieces, and air-dried for 4 days, then ground into mild. The black soybeans were also clean washed and dried for 1 day and ground into mild. The process was done at the Balai Materia Medika, Batu City.

\section{Experimental animals}

Twenty-four rats (Rattus norvegicus) from Wistar strain (age of $10-12$ weeks, weight $85 \pm$ 10 g) obtained from CV. Karunia Jasa Pratama, Malang, Indonesia. The rats placed in a plastic cage with free access to food and drink. Rats were acclimated for a week. After the acclimation, the rats were divided into two groups: normal diet and high-calorie diet (HCD). The normal diet (PT. Comfeed Indonesia) contains 63\% carbohydrate, $3 \%$ fat, $13 \%$ protein, $21 \%$ vitamin and mineral. HCD feed used Hi-Gro Medicated 551 (produced by PT Pokhpand, Indonesia) containing $74 \%$ carbohydrate, $6 \%$ fat, $20 \%$ protein, vitamin, mineral, and $1 \%$ fiber (18). The water in HCD groups was placed with $10 \%$ sucrose.

\section{Induction of diabetes and treatment}

After 30 days of diet manipulation, rats were injected with streptozotocin (STZ) (41910012-3, Bioworld) multiple low doses of $30 \mathrm{mg} / \mathrm{kgBW}$ intraperitoneally [19]. The rats were considered DM if the blood glucose level $\geq 200 \mathrm{mg} / \mathrm{dl}$. DM rats then divided into 8 groups randomly: normal, K(T2DM), $\mathrm{K}+(\mathrm{T} 2 \mathrm{DM}+$ glibenclamide $0.6 \mathrm{mg} / \mathrm{kg}$ BW), P1 (T2DM + black soybean), P2 (T2DM + purple sweet potato), and P3-5 Combination 1-3 (T2DM + combination of black soybean and purple sweet potato in ratio of 1:3, 2:2, and 3:1), respectively. Treatments were given for 30 days. The rats then sacrificed with euthanization (4\% isofluorane), dissected, and the kidney organ was taken and washed using PBS three times. The kidney organ was fixed in $10 \%$ formalin. All research procedures had been approved by the Research Ethics Commission of the University of Brawijaya, Malang with No. App. 878-KEP-UB.

\section{Histological analysis}

Kidney organ fixed in $10 \%$ buffer formalin, dried, and embedded in paraffin. Kidney tissues cut into $5 \mu \mathrm{m}$ thick pieces and stained using hematoxylin-eosin (HE). Kidney slides were observed using a light microscope (CX23, Olympus, Japan). Renal tubular necrosis observation based on count cell in ten different field with $1000 \times$ magnifica- 


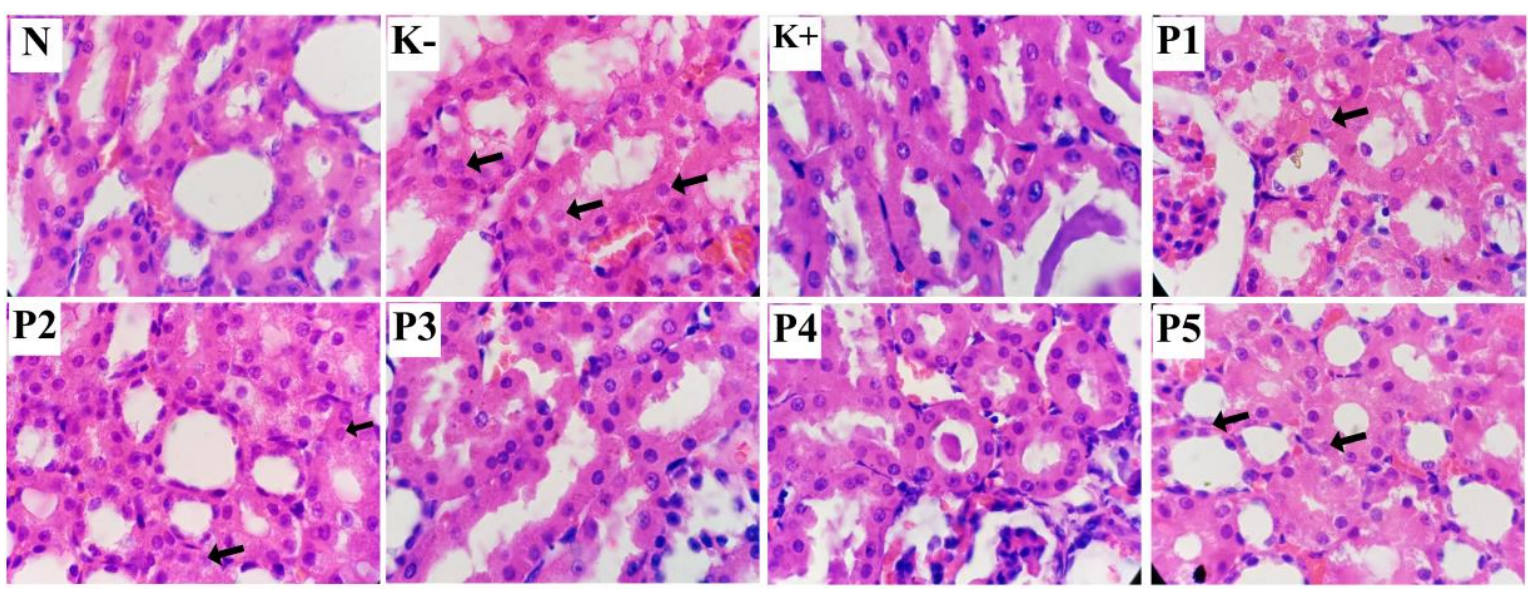

(a)

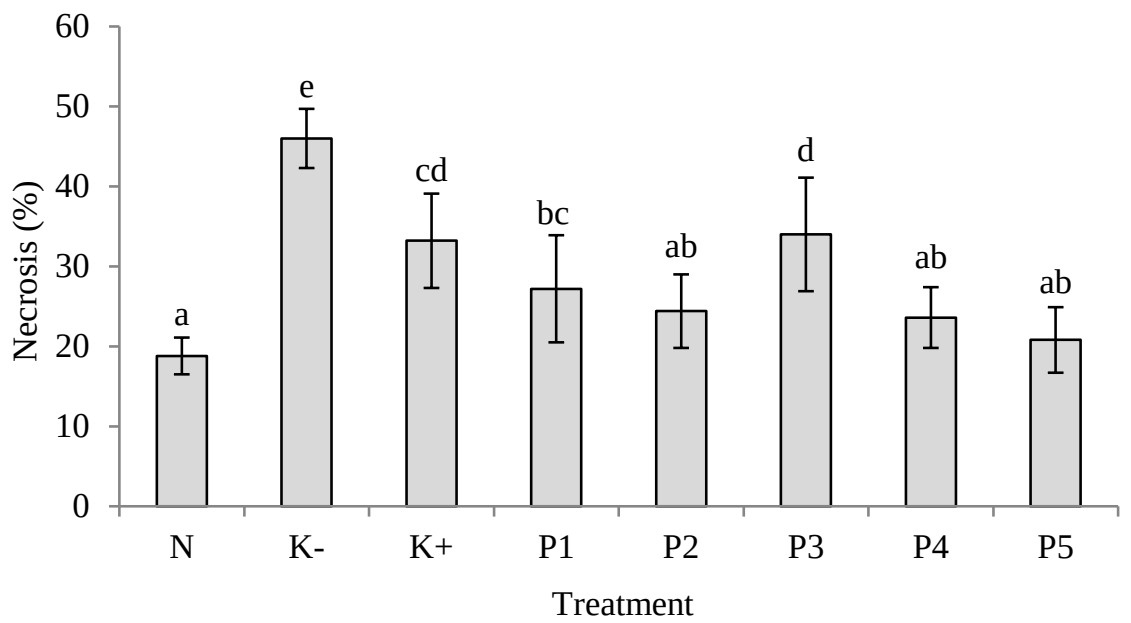

(b)

Figure 1. (a) HE staining in the renal tubular section on normal and experimental groups with 100×. (b) Necrosis percentage on normal and experimental groups. N, normal diet group; K-, T2DM group; K+, T2DM treated with glibenclamide $0,6 \mathrm{mg} / \mathrm{kg}$ BW; P1, T2DM treated with black soybean; P2, T2DM treated with purple sweet potato; P3-P5, T2DM treated with combination of black soybean and purple sweet potato $3: 1,2: 2,1: 3$ respectively. Black arrow showed necrosis of renal tubules cells. The different alphabetic indicated significance $(\mathrm{P}<0.05)$ between groups based on DMRT as a post hoc test.

tion.

\section{Immunohistochemistry analysis}

The expression of Advanced Glycation End Product (AGE) and receptors of AGE-modified proteins (RAGE) in kidney tissues was tested using staining method of immunohistochemistryfluorescence (IHC-F). The primary antibody of rat anti-AGE (No. cat. SC 365154, Abcam, USA), mice anti-RAGE (No. cat. SC-65154, Santa Cruz Biotechnology) and 2\% BSA in the ratio of 1:1:1000, incubated for an hour. Preparations of secondary antibody staining conducted by mixing secondary antibody goat anti-rat IgG Fluorescein Isothiocyanate (FITC) (No. cat. 02-16-06, KPL,
USA), secondary antibody goat anti-mouse IgG Tetramethylrhodamine isothiocyanate (No. cat. Ab6768, Abcam, USA), and 2\% BSA in ratio of 1 $: 1: 1500$ and incubated for an hour. The prepara tions then washed using PBS pH 7.4 three times, dried with tissue paper on the edge, and covered with cover glass. The double staining IHC-F method was observed with a fluorescent microscope (FSX 100, Olympus, Japan) to measure their intensity (intensity/mm).

\section{Statistical analysis}

Data from the observation result of prepara- 


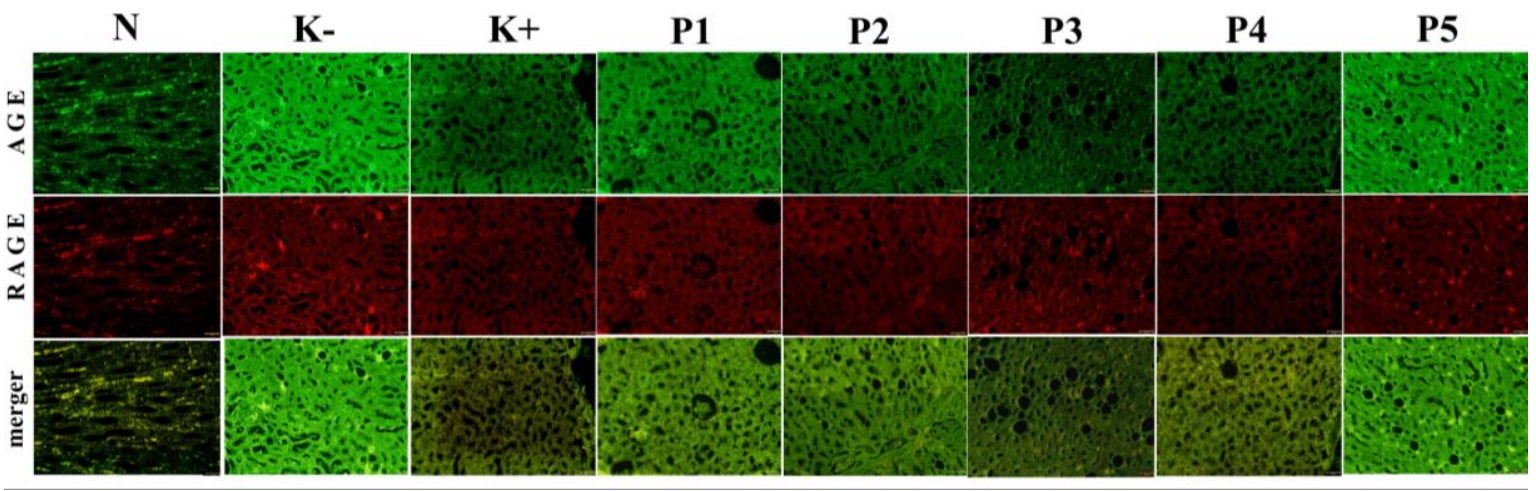

(a)

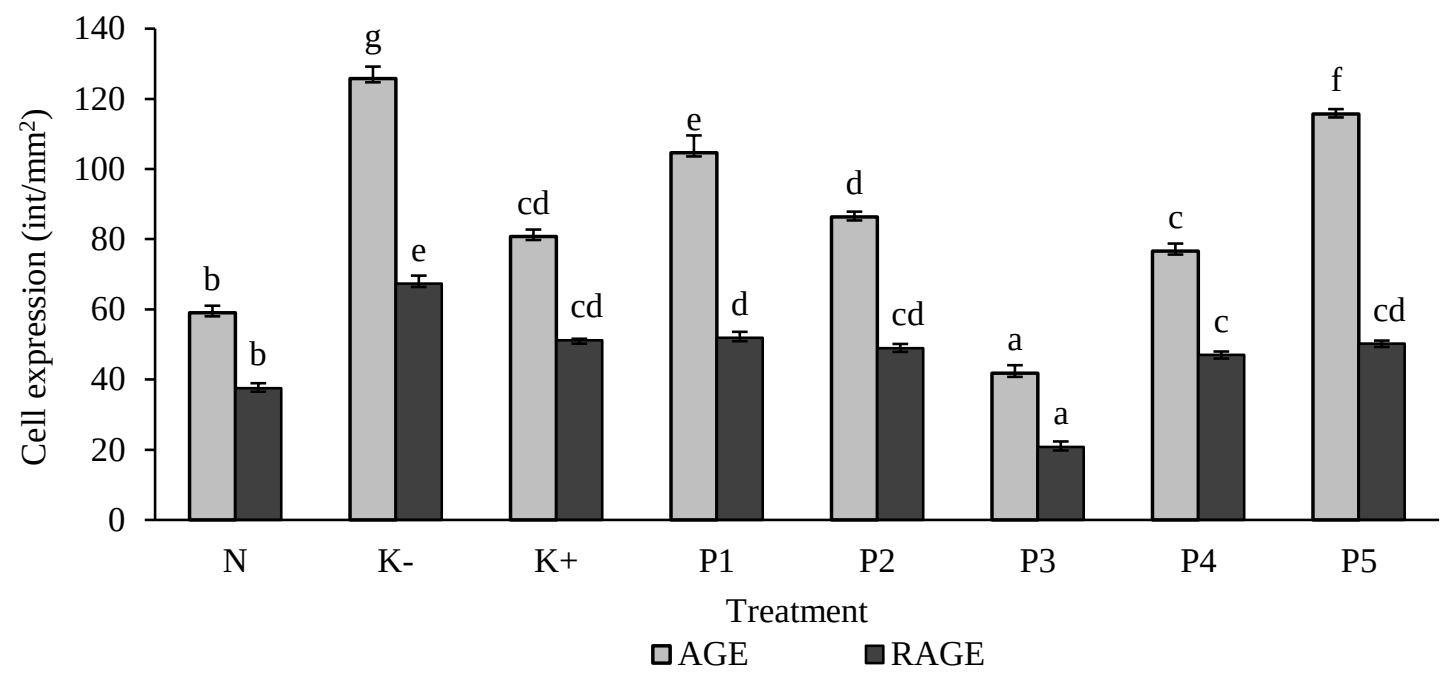

(b)

Figure 1. AGE-RAGE expression in rat after treatment with black soybean, purple sweet potato, and their combination. (a) IHC-F double staining in the renal tubular section on normal and experimental group (observed using a fluorescence microscope, 16× in magnification). (b) AGE-RAGE expression represents as mean \pm standard deviation. $\mathrm{N}$, normal diet group; $\mathrm{K}-$, T2DM group; $\mathrm{K}+, \mathrm{T} 2 \mathrm{DM}+$ glibenclamide $0.6 \mathrm{mg} / \mathrm{kg} \mathrm{BW}$; P1, T2DM treated with black soybean; P2, T2DM treated with purple sweet potato; P3-P5, T2DM treated with combination of black soybean and purple sweet potato $3: 1$, $2: 2,1: 3$ respectively. The different alphabetic indicated significance $(\mathrm{P}<0.05)$ between groups based on DMRT as a post hoc test.

tions stained with HE were analyzed using descriptive qualitative analysis. Data of intensity measurement from IHC-F were analyzed using one-way ANOVA, if significant ( $p<0.05$ ), the analysis continued with a post hoc test of Duncan Multiple Range Test (DMRT).

\section{Results and Discussion}

Comparison of renal tubules necrosis in rats given with black soybean, purple sweet potato, and the combination of both

Kidney preparations stained with HE were used to observe the occurrence of tubules cell ne- crosis in the kidney. Our result indicated that necrosis in tubules of DM group was an increase compared to normal group (Figure 1). Treatment using black soybean, purple sweet potato, or all of the combination indicated that those treatments can decrease necrosis in renal tubules compared to DM groups. The combination of black soybean and purple sweet potato in the ratio of $1: 3$ and 1 : 1 indicated an improvement in tubules condition closer to the normal groups.

Our result suggested that treatments with black soybean, purple sweet potato, or the combination of both reduce tubules cells necrosis on kid- 
ney compared to DM group. Black soybean contains isoflavones genistein and daidzein [20,21]. Jia et al. reported that the rats were given with genistein can attenuate renal fibrosis in STZ-induced diabetic rats. Supplementation with genistein for 8 weeks also increased endogenous antioxidant superoxide dismutase and decreased lipid peroxidase and malondialdehyde (MDA) [22]. Kim and Lim (2013) also reported that supplementation on early stage of diabetic-induced renal damage can decrease level of inflammation markers such as nuclear factor kappa B (NFkB), tumor necrosis factor $\alpha$ (TNF- $\alpha)$, dan cyclooxygenase-1 (COX-1) [23]. Genistein is a group of phytoestrogens that plays a role as a specific inhibitor for $\alpha$-glucosidase. $\alpha$-glucosidase is an enzyme which has the function in carbohydrate digestion. The inhibition of the enzyme causes a delay in the termination of disaccharide chain into monosaccharide (glucose) thus reducing the absorption of glucose in the small intestine [20, 21, 24].

\section{Comparison of AGE and RAGE expression on rats given with black soybean, purple, or the combination of both}

Our results indicated that AGE and RAGE expression in DM was significantly increased compared to normal group (Figure 2a and b). Black soybean, purple sweet potato, or the combination of both were able to significantly decrease the intensity of AGE and RAGE expression compared to those in the DM group (Figure 2a). The combination of black soybean and purple sweet potato in the ratio of $2: 2$ decrease AGE and RAGE intensity closer to normal group. Interestingly, the combination of black soybean and purple sweet potato in the ratio of $1: 3$ decreased the expression of AGE and RAGE lower than the normal group (Figure 2b). Our result suggested that the combination of black soybean and purple sweet potato could be used as a candidate of alternative treatment for T2DM through the decrease of AGE and RAGE expression in kidney.

Type 2 diabetes mellitus is a progressive and degenerative disease marked with a blood glucose level of more than $\geq 200 \mathrm{mg} / \mathrm{dL}$ and caused by insulin resistance. Hyperglycemia causes an increase of AGE-RAGE, then affecting the development of kidney damage formation [10]. Our finding showed DM rats had the highest AGE-RAGE expression in kidney tubules compared to treatment groups. The high expression of AGE-RAGE indicated that there was damage in the kidney tubules. The result was supported by observation on kidney histological changed. The histology of kidney showed there were many cells under necrosis condition.

AGE-RAGE accumulation mediated diabetic nephropathy since the binding between AGE and RAGE increase reactive oxygen species production lead to oxidative stress status [5]. ROS stimulate the overexpression of pro-sclerotic growth factors such as tumor growth factor $\beta$ (TGF $\beta$ ) and connective tissue growth factor (CTGF). In line with our finding, the percentage of necrosis on the proximal tubule of DM rats was higher than nondiabetes rats [25]. Damage on tubules characterizes pyknosis, karyolysis, and necrosis on the brush border. The binding of AGE-RAGE also induces the activation of the transcription factor of NFB, and mitogen-activated protein kinase played a role in the change of phenotype and cell function [7].

Our research result indicated that glibenclamide decreased the AGE-RAGE expression compared to the DM group. Glibenclamide is one of synthesis drug includes in sulphonylurea group. Glibenclamide works by inhibiting ATP-sensitive potassium canal in pancreatic -cells. The inhibition causes the occurrence of calcium canal depolarization thus the intracellular calcium level of pancreatic -cells increases and stimulates insulin production [26]. However, prolonged use of synthesis drug could have side effects for the body. Continuous use of glibenclamide could cause damage in pancreatic-cells due to overstimulation for insulin production [24]; therefore, an alternative treatment from herbal is needed that to reduce the side effects of treatment.

Soybean is also contained protein derivatives, such as glycine and arginine that play an essential role in the secretion of glucagon and insulin by the pancreas [20]. Genistein reported to be able to decrease malondialdehyde levels in the kidney, which is the end product of oxidative stress [21]. Diet using genistein supplement in a dose of 600 $\mathrm{mg} / \mathrm{kg} \mathrm{BW}$ also indicated a decrease in glucose level [21]. The improvement in glucose absorption in the intestines and free radical have ability to alleviate AGE-RAGE expression in kidney cells.

Both black soybean and purple sweet potato 
contain anthocyanin. Anthocyanin is a derivative compound of flavonoid that water soluble and has reddish or purple color pigment [13]. Anthocyanin plays an essential role as an inhibitor of $\alpha$-amylase, maltase, and sucrose [27], thus it reduces the glucose level. Anthocyanin also has activities as an antioxidant since one of its function is as a ROS free radical scavenger [28], leading to the decrease of AGE and RAGE. Anthocyanin was reported to lowering blood glucose level by improving the activity of glucose transporter 4 (GLUT4) in muscles and adipose tissues [28].

\section{Conclusion}

The combination of black soybean and purple sweet potato was able to reduce the AGE, RAGE, and renal necrosis in DM rats. The combination suggested to improve AGE, RAGE, and necrosis was $2: 2$. Consumption of black soybean and purple sweet potato is expected to become a simple and cheap functional food to be used as alternative therapy for DM. However, further research is required before the combination of both can be applied to human by giving appropriate dose recommendation.

\section{Acknowledgment}

The research was funded by the Ministry of Research Technology and Higher Education with research contract no. 3.4.8/UN32.14/LT/2017.

\section{References}

1. Skovsø S (2014) Modeling type 2 diabetes in rats using high fat diet and streptozotocin. Journal of Diabetes Investigation 5 (4): 349-358. doi: 10.1111/jdi.12235.

2. Hajiaghaalipour F, Khalilpourfarshbafi M, Arya A (2015) Modulation of glucose transporter protein by dietary flavonoids in type 2 diabetes mellitus. International Journal of Biological Sciences 11 (5): 508-524. doi: 10.7150/ijbs.11241.

3. Martínez-Castelao A, Navarro-González J, Górriz J, de Alvaro F (2015) The Concept and the epidemiology of diabetic nephropathy have changed in recent years. Journal of Clinical Medicine 4 (6): 1207-1216. doi: 10.3390/jcm4061207.

4. World Health Organization (2016) About diabetes: Complications of diabetes. Geneva, WHO.

5. Yamagishi S, Matsui T (2010) Advanced glycation end products, oxidative stress and diabetic nephropathy. Oxidative Medicine and Cellular Longevity 3 (2): 101-108. doi: 10.4161/oxim.3.2.11148.

6. Zini E, Benali S, Coppola L et al. (2014) Renal morphology in cats with diabetes mellitus. Veterinary Pathology 51 (6): 1143-
1150. doi: 10.1177/0300985813516645.

7. Al-Qattan KK, Mansour MH, Thomson M, Ali M (2016) Garlic decreases liver and kidney receptor for advanced glycation end products expression in experimental diabetes. Pathophysiology 23 (2): 135-145. doi: 10.1016/j.pathophys.2016.02.003.

8. Bohlender JM, Franke S, Stein G, Wolf G (2005) Advanced glycation end products and the kidney. American Journal Physiological Renal Physiology 289 645-659. doi: 10.1152/ajprenal.00398.2004.

9. Daroux M, Grossin N, Boulanger E (2012) AGE, RAGE, and diabetic nephropathy. US Endocrinology 08 (02): 98-103. doi: 10.17925/USE.2012.08.02.98.

10. Watson AMD, Gray SP, Jiaze L et al. (2012) Alagebrium reduces glomerular fibrogenesis and inflammation beyond preventing RAGE activation in diabetic apolipoprotein e knockout mice. Diabetes 61 (8): 2105-2113. doi: 10.2337/db11-1546.

11. Patwardhan B (2005) Traditional medicine: Modern approach for affordable global health. https://www.who.int/intellectualproperty/studies/B.Patwardhan2.pdf?ua=1. Accessed date: May 2019. Pp 1-172.

12. Koh K, Youn JE, Kim H-S (2014) Identification of anthocyanins in black soybean (Glycine max (L.) Merr.) varieties. Journal of Food Science and Technology 51 (2): 377-381. doi: 10.1007/s13197-011-0493-y.

13. Sivamaruthi BS, Kesika P, Subasankari K, Chayaisut C (2018) Beneficial effects of anthocyanins against diabetes mellitus associated conseuence- A mini review. Asian Pacific Journal of Tropical Biomedicine 8 (10): 471-477. doi: 10.4103/22211691.244137.

14. Kano M, Takayanagi T, Harada $\mathrm{K}$ et al. (2005) Antioxidative activity of anthocyanins from purple sweet potato, Ipomoera batatas cultivar Ayamurasaki. Bioscience, Biotechnology, and Biochemistry 69 (5): 979-988. doi: 10.1271/bbb.69.979.

15. Lee CL, Lee SL, Chen CJ et al. (2016) Characterization of secondary metabolites from purple Ipomoea batatas leaves and their effects on glucose uptake. Molecules 21 (6): 745-758. doi: 10.3390/molecules21060745.

16. Gofur A, Witjoro A, Widya Ningtiyas E et al. (2018) The ameliorative effect of black soybean and purple sweet potato to improve sperm quality through suppressing reactive oxygen species (ROS) in type 2 diabetes mellitus rat (Rattus novergicus). Science Asia 44 (5): 303. doi: 10.2306/scienceasia15131874.2018.44.303.

17. Nimse SB, Pal D (2015) Free radicals, natural antioxidants, and their reaction mechanisms. RSC Advances 5 (35): 27986 28006. doi: 10.1039/C4RA13315C.

18. Lestari SR, Djati MS, Rudijanto A, Fatchiyah F (2015) PPARy expression by rambutan peel extract in obesity rat model-induced high-calorie diet. Asian Pacific Journal of Tropical Biomedicine 5 (10): 852-857. doi: 10.1016/j.apjtb.2015.01.030.

19. Zhang M, Lv XY, Li J et al. (2008) The Characterization of 
high-fat diet and multiple low-dose streptozotocin induced type 2 diabetes rat model. Experimental Diabetes Research (2008): 1-9. doi: 10.1155/2008/704045.

20. Ahmad A, Hayat I, Arif S et al. (2014) Mechanisms involved in the therapeutic effects of soybean (Glycine max). International Journal of Food Properties 17 (6): 1332-1354. doi: 10.1080/10942912.2012.714828.

21. Kim MJ, Lim Y (2013) Protective effect of short-term genistein supplementation on the early stage in diabetes-induced renal damage. Mediators of Inflammation (2013): 1-14. doi: 10.1155/2013/510212

22. Jia Q, Yang R, Liu X-F et al. (2019) Genistein attenuates renal fibrosis in streptozotocin-induced diabetic rats. Molecular Medicine Reports 19 (1): 423-431. doi: 10.3892/mmr.2018.9635.

23. Kim MJ, Lim Y (2013) Protective effect of short-term genistein supplementation on the early stage in diabetes-induced renal damage. Mediators of Inflammation (2013): 1 - 14. doi: 10.1155/2013/510212

24. Choudhury H, Pandey M, Hua CK et al. (2018) An update on natural compounds in the remedy of diabetes mellitus: A sys- tematic review. Journal of Traditional and Complementary Medicine 8 (3): 361-376. doi: 10.1016/j.jtcme.2017.08.012.

25. Alipin K, Sari EP, Setiawati T et al. (2017) Kidney histology in streptozotocin-induced diabetic male Wistar rats treated with combined extract of temulawak rhizome and belimbing wuluh fruit. Nusantara Bioscience 9 (3): 312-317. doi: 10.13057/nusbiosci/n090312.

26. Rai A, Eapen C, Prasanth VG (2012) Interaction of herbs and glibenclamide: A review. ISRN Pharmacology 2012 1-5. doi: 10.5402/2012/659478.

27. Reverri E, Randolph J, Steinberg F et al. (2015) Black beans, fiber, and antioxidant capacity pilot study: Examination of whole foods vs. functional components on postprandial metabolic, oxidative stress, and inflammation in adults with metabolic syndrome. Nutrients 7 (8): 6139-6154. doi: 10.3390/nu7085273.

28. Belwal T, Nabavi S, Nabavi S, Habtemariam S (2017) Dietary anthocyanins and insulin resistance: When food becomes a medicine. Nutrients 9 (10): 1111-1132. doi: 10.3390/nu9101111. 
This page is intentionally left blank. 